Ian White MBBS FCA (SA) FRCPC, Greg Doak MD FRCPC

\title{
Outcomes analysis can provide new directions for the management of patients undergoing lumbar spine surgery
}

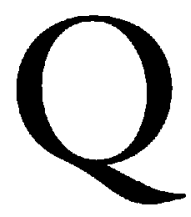

UALITY of care in modern medicine is based on the thesis that decisions are made on the best available evidence. Anesthesia has become very safe. In the past, assessment of the quality of anesthetic services centered around anesthesia related mortality. In a series of articles covering 1957 to 1987 , Harrison ${ }^{1}$ showed a 2.5 to 3 fold decrease in anesthesia mortality from 0.19 deaths per 1000 anesthetics to 0.07 deaths per 1000 . In the 1980s it became clear that if anesthesiologists were to improve care, they needed to study anesthesia related morbidity. In 1986 Cohen $e t a l^{2}$ showed that the risk of complications increased over time. The risk of a postoperative recovery room complication rate increased from $3.1 \%(1975-1978)$ to $5.9 \% \quad(1979-83)$. Subsequently, "The Canadian four-centre study of anesthesia outcomes" 3 suggested that minor adverse events, particularly those of concern to the patient, should be the next focus for quality improvement in anesthesia.

In this issue of the Journal, Manninen et al..$^{4}$ assess the incidence and characteristics of early post-operative complications in patients following neurosurgical procedures. The study followed 486 patients. At least one complication occurred in $54.5 \%$ of the patients. Overall, there was a high incidence of early post-operative complications, mostly due to nausea and vomiting, compared with other studies. ${ }^{5}$ However, of greatest concern is the high overall incidence of complications in the patients undergoing spinal surgery. One hundred and sixty nine patients underwent spinal surgical procedures. In 12 patients in the cervical group and in two in the thoraco-lumbar group the lungs were ventilated in the post-operative period and these patients were excluded from follow-up.

In the remaining 155 patients, an alarming 138 patients $(89 \%)$ suffered complications in the post- operative period. While most, $78(50 \%)$, of the patients suffered from nausea and vomiting, more ominously, 20 patients (13\%) experienced respiratory complications in the post-operative period, including aspiration. Nineteen patients experienced airway trauma, $16(84 \%)$ had been placed in the prone position during surgery. These findings suggest a rethinking of our approach to anesthesia for lumbar spinal surgery. Most centres rely on general inhalational anesthesia for these procedures. Is there a better approach?

Patients undergoing spinal cord surgery may have been suffering nerve root irritation for some time before surgery. Repeated noxious stimuli induce neuroplastic changes. The population of patients with chronic back pain, therefore, often present for spinal cord surgery after the failure of long periods of conservative treatment and have developed chronic back pain syndromes due to these neuroplastic changes. Accordingly their analgesic requirements are increased both during the operative and post-operative periods. In the current study, there was an increased use of intraoperative and post-operative opioid analgesics in this group of patients compared with the other patients in the study. Obviously, strategies to decrease the use of opioid analgesics and to reduce the pain experienced by this "high risk" group of patients could improve anesthesia outcome.

Spinal opioids act pre-synaptically to inhibit release of nociceptive mediators. In the setting of established "wind-up", this pre-synaptic inhibition may be insufficient to prevent ongoing post-synaptic excitation of the sensitized spino-thalamic neuron. In some clinical situations higher doses of opioids are required to suppress this post-synaptic activation. Under these conditions, blockade of the $\mathrm{N}$-methyl-D-aspartate (NMDA) receptor is much more effective at suppress-

Address correspondence to: Dr. Ian White MBBS FCA(SA) FRCPC, Associate Professor University of Manitoba, and Section Head Neuroanaesthesia, L2035, Saint Boniface General Hospital, 409 Tache Avenue, Winnipeg, MB, Canada R2H 2 A6 
ing established windup. ${ }^{6,7}$ Opioid receptor activation itself may facilitate protein kinase C mediated NMDA receptor activation. The resulting calcium influx initiates a series of intracellular events (part of the wind-up phenomenon), one of which is a functional uncoupling of the opioid receptors resulting in opioid tolerance. ${ }^{8}$ Blockade of the NMDA receptor may be critical in providing adequate analgesia, reducing the effects of spinal neuroplasticity, and reversing opioid tolerance. To this end ketamine and dextromethorphan are clinically available NMDA receptor blockers.

Strategies that will provide protection against additional stimulation of the post-synaptic neurone by presynaptic receptor blockade of both opioid and NMDA receptors would be expected to improve outcomes.

The above provide theoretical grounds for a change in practice. What is the clinical evidence for a change in practice? Either general or regional anesthesia has been shown to be safe for patients undergoing lumbar spine surgery. ${ }^{9}$ Using regional anesthesia for these procedures confers advantages in reduced blood loss and improved operating conditions by reducing venous blood loss in the operative field. ${ }^{10}$ In two uncontrolled large retrospective studies spinal anesthesia has been shown to be at least as good or better than general anesthesia in patients undergoing lumbar spine surgery. ${ }^{11}$ Administration of preemptive spinal opioid agonists at the time of the administration of spinal local anesthetic may, therefore, prevent the consequences of noxious stimulus-induced neuroplasticity at the time of surgery.

An increase in postoperative morbidity has been observed ${ }^{12}$ in patients undergoing lumbar laminectomy. Adequate pain relief hastens rehabilitation and the incidence of chronic pain is decreased. The degree of postoperative pain relief with epidural opioids is superior to that achieved with intramuscular and intravenous patient controlled analgesia. ${ }^{12}$ The study by Kundra shows that the use of preemptive epidural morphine is superior to epidural morphine given postoperatively for pain relief after lumbar laminectomy.

This study by Manninen et al. raises many questions. They show a high incidence of complications following spinal surgery, which is of concern. Well constructed prospective trails comparing spinal anesthesia to general anesthesia for lumbar spine surgery suggests itself.

\section{L'analyse de l'évolution postopératoire : nou- velles orientations pour}

\section{la chirurgie de la colonne lombaire}

L'idée de base de la qualité des soins de la médecine moderne veut que les décisions soient prises à partir des meilleures données diponibles. L'anesthésie est devenue très sûre. Dans le passé, l'évaluation de la qualité des soins anesthésiques était centrée sur la mortalité reliée à l'anesthésie. Dans une série d'articles produits entre 1957 et 1987, Harrison ${ }^{1}$ a montré un taux de mortalité reliée à l'anesthésie de 2,5 à 3 fois plus bas passant de 0,19 à 0,07 par 1000 anesthésies. Dans les années 1980, il est devenu évident que si les anesthésiologistes voulaient améliorer les soins, ils devaient étudier la morbidité reliée à l'anesthésie. En 1986, Cohen et coll. ${ }^{2}$ ont montré que le risque de complications postopératoires a augmenté avec le temps, le taux passant de 3,1\% (1975-1978) à 5,9\% (1979-1983) observé en salle de réveil. Par la suite, dans le but d'améliorer la qualité de l'anesthésie, «The Canadian four-centre study of anesthesia outcomes» ${ }^{3}$ a proposé que les effets secondaires mineurs, en particulier ceux qui préoccupent le patient, deviennent le prochain centre d'intérêt.

Dans le présent numéro du Journal, Manninen et coll. ${ }^{4}$ évaluent l'incidence et les caractéristiques des complications postopératoires précoces chez des patients qui ont subi une neurochirurgie. L'étude a porté sur 486 patients. Une complication au moins est survenue chez $54,5 \%$ des patients. Globalement, par rapport à d'autres études, il y a une incidence élevée de complications postopératoires précoces, surtout des nausées et des vomissements. ${ }^{5}$ Cependant, lorsqu'il s'agit de patients subissant une chirurgie de la colonne vertébrale, cette situation inquiète davantage. Cent soixante-neuf patients ont subi une chirurgie de la colonne. Douze patients du groupe de chirurgie cervicale et deux du groupe de chirurgie thoraco-lombaire ont eu besoin de ventilation pulmonaire après l'intervention et ont été exclus du suivi.

Chez les 155 patients restants, un nombre alarmant de patients $(138,89 \%)$ ont souffert de complications postopératoires. Tandis que la plupart des patients, 78 (50\%), ont eu des nausées et des vomissements, 20 patients ( $13 \%)$, ce qui est plus inquiétant, ont présenté des complications respiratoires postopératoires, y compris l'aspiration. Dix-neuf patients ont subi un traumatisme des voies aériennes dont $16(84 \%)$ avaient été placés en décubitus ventral pendant la chirurgie. Ces constatations incitent à repenser la façon d'aborder 
l'anesthésie lors d'une chirurgie de la colonne lombaire. Actuellement, dans la plupart des centres, on a recours à l'anesthésie générale par inhalation. Y a-t-il une meilleure méthode ?

Prenons le cas de patients qui subissent une chirurgie de la moelle épinière. Ils ont pu souffrir d'irritation des racines nerveuses pendant quelque temps avant la chirurgie. Des stimuli nocifs répétés induisent en effet des changements de neuroplasticité qui peuvent entrainer des douleurs chroniques au dos. Les sujets qui en sont atteints, sont souvent admis pour une chirurgie de la moelle épinière à la suite de l'échec de traitements conservateurs à long terme. Par conséquent, leurs besoins d'analgésiques augmentent pendant et après l'opération. Dans l'étude en question, on a utilisé davantage d'analgésiques opiacés pendant et après l'intervention pour ce groupe de patients comparés aux autres patients de l'étude. À l'évidence, des stratégies pour diminuer l'usage des analgésiques opiacés et pour réduire la douleur ressentie par ce groupe de patients à "haut risque" pourraient améliorer les résultats de l'anesthésie.

Les opiacés intrarachidiens agissent de façon présynaptique pour empêcher la libération de médiateurs nociceptifs. Dans le cas d'un «wind-up» établi, cette inhibition présynaptique peut être insuffisante pour empêcher l'excitation postsynaptique déjà présente du neurone sensitif spinothalamique. Certaines situations cliniques exigent de plus grandes doses d'opiacés pour supprimer cette activation postsynaptique. Le blocage du récepteur N-méthyl-D-aspartate (NMDA) est alors beaucoup plus efficace pour supprimer le "wind-up" établi. ${ }^{6,7}$ L'activation du récepteur d'opiacés lui-même peut faciliter l'activation du récepteur NMDA par la médiation de la protéine kinase $\mathrm{C}$. Le flux calcique qui en résulte provoque une série d'événements intracellulaires, donc une partie du phénomène de "windup", l'un d'entre eux étant un découplement fonctionnel des récepteurs opiacés qui induit une tolérance aux opiacés. La kétamine et le dextrométhorphane sont les inhibiteurs du récepteur NMDA offerts en clinique à cette fin.

On pourrait s'attendre que les stratégies qui assureront une protection contre une stimulation additionnelle de la portion postsynaptique du neurone par l'inhibition du récepteur présynaptique des récepteurs opiacés et NMDA vont améliorer les résultats.

Ce qui précède fournit des bases théoriques à un changement de pratique. Mais qu'est-ce qui constitue une évidence clinique justifiant un changement de pratique ? On a montré que tant l'anesthésie générale que régionale est sûre pour les patients qui subissent une chirurgie de la colonne lombaire.' L'emploi de l'anesthésie régionale pour ces interventions permet de réduire les pertes sanguines et d'améliorer les conditions de l'opération par la réduction de perte de sang veineux dans le champ opératoire. ${ }^{10}$ Lors de deux grandes études rétrospectives non contrôlées, on a démontré que la rachianesthésie est au moins aussi bonne ou meilleure que l'anesthésie générale chez des patients qui ont subi une chirurgie de la colonne lombaire. " L'administration préventive d'agonistes d'opiacés rachidiens au moment de la rachianesthésie peut donc éliminer les conséquences des changements de neuroplasticité induits par un stimulus douloureux lors de la chirurgie.

On a observé une hausse de la morbidité postopératoire ${ }^{12}$ chez des patients qui subissent une laminectomie lombaire. Un soulagement adéquat de la douleur accélère la réadaptation et fait diminuer l'incidence de la douleur chronique. Le degré du soulagement de la douleur postopératoire avec les opiacés périduraux est supérieur à celui qu'on obtient avec un analgésique intramusculaire et intraveineux, ce dernier contrôlé par le patient. ${ }^{12}$ L'étude de Kundra souligne que l'emploi de morphine péridurale préventive est supérieur à celui de la morphine péridurale administrée après l'opération pour soulager la douleur de la laminectomie lombaire.

L'étude de Manninen et coll. soulève bien des questions. Les auteurs ont montré une forte incidence de complications à la suite d'une chirurgie de la colonne, ce qui est préoccupant. Des essais cliniques prospectifs bien construits comparant la rachianesthésie et l'anesthésie générale pour la chirurgie de la colonne lombaire deviennent évidents.

\section{References}

1 Harrison GG. Death due to anaesthesia at Groote Schuur Hospital, Cape Town - 1956-1987. Part I. Incidence. S Afr Med J 1990; 77: 412-5.

2 Cohen $M M$, Duncan PJ, Pope WD, Wolkenstein C. A survey of 112,000 anaesthetics at one teaching hospital (1975-83). Can Anaesth Soc J 1986; 33: 22-31.

3 Coben MM, Duncan PG, Pope WD, et al. The Canadian four-centre study of anaesthesia outcomes. II. Can outcomes be used to assess the quality of anaesthesia care? Can J Anaesth 1992; 39: 430-9.

4 Manninen PH, Raman SK, Boyle $K$, El-Bebeiry $H$. Early postoperative complications following neurosurgical procedures. Can J Anesth 1999; 46: 7-14.

5 Coben MM, Duncan PG, DeBoer DP, Tweed WA. The postoperative interview: assessing risk factors for nausea and vomiting. Anesth Analg 1994; 78: 7-16.

6 Dickenson AH. A cure for windup: NMDA receptor antagonists as potential analgesics. Trends Pharmacol Sci $1990 ; 11: 307-9$. 
7 Coderre TJ, Katz J, Vaccarino AL, Melzack R.

Contribution of central neuroplasticity to pathological pain: review of clinical and experimental evidence. Pain 1993; 52: 259-85.

8 Mayer DJ, Mao J, Price DD. The development of morphine tolerance and dependence is associated with translocation of protein kinase C. Pain 1995; 61: 365-74.

9 Jellish WS, Thalji $Z$, Stevenson $K$, Shea J. A prospective randomized study comparing short- and intermediateterm perioperative outcome variables after spinal or general anesthesia for lumbar disc and laminectomy surgery. Anesth Analg 1996; 83: 559-64.

10 Horlocker TT, Cucchiara RF, Ebersold MJ. Vertebral column and spinal cord surgery. In: Cucchiara RF, Michenfelder JD (Eds.). Clinical Neuroanesthesia. New York: Churchill Livingstone, 1990: 325-50

11 Ditzer JW, Dumke PR, Harrington J, Fox JD. Should spinal anaesthesia be used for surgery for herniated intervertebral disk? Anesth Analg 1959; 38: 118-24.

12 Kundra P, Gurani A, Bhattacharya A. Preemptive epidural morphine for postoperative pain relief after lumbar larninectomy. Anesth Analg 1997; 85: 135-8. 